\title{
Effect of orally administered glutathione on glutathione levels in some organs of rats: role of specific transporters
}

\author{
BY FABIO FAVILLI, PATRIZIA MARRACCINI, TERESA IANTOMASI \\ AND MARIA T. VINCENZINI*
}

Dipartimento di Scienze Biochimiche, Università di Firenze, 50134 Firenze, Italy

(Received 7 May 1996 - Revised 28 January 1997 - Accepted 31 January 1997)

\begin{abstract}
The present study reports data on absorption of orally administered glutathione (GSH) in rat jejunum and in other organs, and the possible role of specific transport systems of GSH and $\gamma$ glutamyltranspeptidase (EC 2.3.2.1; $\gamma$-GT) activity. GSH levels were measured simultaneously in various organs after oral GSH administration to untreated rats and rats treated with L-buthionine sulfoximine (BSO) or acivicin ( $\left.\mathrm{AT}_{125}\right)$. BSO selectively inhibits GSH intracellular synthesis and $\mathrm{AT}_{125}$ is a specific inhibitor of $\gamma$-GT activity. GSH levels were also measured after oral administration of an equivalent amount of the constituent amino acids of GSH to untreated and BSO-treated rats. Significant increases in GSH levels were found in jejunum, lung, heart, liver and brain after oral GSH administration to untreated rats. GSH increases were also obtained in all organs, except liver, when GSH was administered to rats previously GHS-depleted by treatment with BSO. The analysis of all results allowed us to distinguish between the increase in GSH intracellular levels due to intact GSH uptake by specific transporters, and that due to GSH degradation by $\gamma$-GT activity and subsequent absorption of degradation products with intracellular resynthesis of GSH; both these mechanisms seemed to be involved in increasing GSH content in heart after oral GSH administration. Jejunum, lung and brain took up GSH mostly intact, by specific transport systems, while in liver GSH uptake occurred only by its breakdown by $\gamma$-GT activity followed by intracellular resynthesis.
\end{abstract}

Glutathione: Intestine: $\boldsymbol{\gamma}$-Glutamyltranspeptidase

A number of observations have shown that dietary glutathione (GSH) can be absorbed intact from the intestinal lumen and that orally administered GSH increases plasma GSH concentration (Hagen et al. 1990; Jones, 1995). It has also been demonstrated in mice that oral GSH administration can increase the GSH concentration in some tissues but only after GSH depletion (Aw et al. 1991). In fact, intestinal absorption of exogenous GSH can be very important, both in detoxification mechanisms where the intestinal epithelium is involved and in supplying plasma as well as other tissues with GSH and cysteine. In some pathological and physiological conditions intracellular GSH synthesis is not able to maintain high levels of this tripeptide in intestine and in other organs of many species, including man, i.e. metabolic disorders, stress oxidative disorder, malnutrition, Crohn's disease, Parkinson's disease, Alzheimer's disease, human immunodeficiency virus infection and ageing (Meister \& Larsson, 1989; Bray \& Taylor, 1993; Stio et al. 1993; Favilli et al. 1994; Grisham, 1994; Iantomasi et al. 1994; Castagna et al. 1995); therefore oral therapeutic administration of GSH has been suggested, even though many studies on the ability of exogenous GSH to increase tissue GSH reported in the literature are contradictory. Four different mechanisms of GSH uptake through the membranes have been

\footnotetext{
* For reprints.
} 
suggested (Martensson et al. 1990); however, the main pieces of experimental evidence regarding GSH transport are: (1) the breakdown of GSH into its constituent amino acids by specific membrane enzymes, $\gamma$-glutamyltranspeptidase $(E C 2.3 .2 .1 ; \gamma$-GT) and dipeptidase, followed by their transport and subsequent intracellular GSH resynthesis. This is a very complex mechanism involving the enzymes of the $\gamma$-glutamyl cycle, which includes the GSH synthesis enzymes as well as the consumption of ATP; (2) the uptake of intact GSH by specific transporters, a much faster mechanism, which consumes less energy. Although both processes allow the uptake of exogenous GSH, they are independent and may occur simultaneously (Vincenzini et al. 1992). In various cells specific transport systems have been identified (Aw et al. 1991). In particular, in jejunum of rat and rabbit a $\mathrm{Na}^{+}-\mathrm{in}$ dependent facilitated transport system for GSH and GSH-S-conjugates in brush-border membranes (Vincenzini et al. 1988), and a GSH $\mathrm{Na}^{+}$-dependent system in basolateral membranes (Lash et al. 1986) have been characterized. Considering these previous results and the possibility of oral GSH therapy we performed the present study to assess the efficacy of oral GSH administration in increasing the GSH content of various organs under different experimental conditions. Moreover, the physiological roles of specific GSH transport systems and $\gamma$-GT activity in GSH uptake by intestinal epithelium and other tissues were considered. For this purpose we measured GSH levels simultaneously in jejunum, ileum, liver, lung, heart and brain after oral GSH administration to untreated rats and rats treated with $\mathrm{L}$-buthionine sulfoximine $(\mathrm{BSO})$ or acivicin $\left(\mathrm{AT}_{125}\right)$. Administration of BSO to rats specifically inhibits endogenous synthesis of GSH and selectively decreases intracellular GSH content. This inhibitor does not demonstrate toxicity in animals (Martensson et al. 1991); administration of BSO to rats for long periods leads to marked structural changes in various organs (Martensson et al. 1990). In the present study BSO was given only for $1 \mathrm{~d}$ and it was used to evaluate the absorption of intact GSH by transport systems, since the regulator enzyme of GSH endogenous synthesis, $\gamma$-glutamylcysteine synthetase ( $E C$ 6.3.2.2; $\gamma$-GCS) is inhibited by BSO.

$\mathrm{AT}_{125}$ administration inhibits the activity of $\gamma$-GT, the only known enzyme that can break down the $\gamma$-glutamyl linkage of GSH; therefore we were able to verify the involvement of this enzyme, as well as of GSH degradation products, in GSH uptake. This is the first time that $\mathrm{AT}_{125}$ has been used to investigate the role of $\gamma$-GT activity in increasing GSH in various organs after oral GSH administration. $\mathrm{AT}_{125}$ has minimal toxicity when it is given in vivo (Hill et al. 1985).

GSH levels were also measured in some organs after oral administration of the three constituent amino acids of GSH (Glu, Cys, Gly) to untreated and BSO-treated rats. The comparison of all results obtained under these different experimental conditions allowed us to assess the usefulness of oral GSH supplementation in enhancing tissue GSH, and to determine if the GSH increases measured were due to intact GSH uptake by transport systems and/or by its $\gamma$-GT degradation and subsequent GSH intracellular resynthesis. It is also possible to propose physiological roles for both $\gamma$-GT and transporter activities in maintaining an inter-organ cycle of GSH.

\section{MATERIALS AND METHODS}

\section{Materials}

L-Buthionine-S, $R$-sulfoximine (BSO), $\mathrm{AT}_{125}, \mathrm{GSH}$, glutamate, cysteine and glycine were obtained from Sigma Chemical Corp (St Louis, MO, USA). The dye reagent concentrate for the determination of protein was obtained from Bio Rad Laboratories S.r.l. (Milan, 
Italy). All other chemicals used were reagent grade and were obtained from commercial sources.

\section{Animal treatments}

Rats (male, 50-80 g Sprague-Dawley; Nossan Correzzano, Italy) were fed on a complete standard diet containing about $760 \mathrm{~g}$ yellow maize $/ \mathrm{kg}, 200 \mathrm{~g}$ wheat gluten $/ \mathrm{kg}, 30 \mathrm{~g}$ $\mathrm{CaCO}_{3} / \mathrm{kg}$ and $10 \mathrm{~g} \mathrm{NaCl} / \mathrm{kg}$, and were provided water ad libitum for 1 week before the different treatments. The rats were fasted for $24 \mathrm{~h}$ before the beginning of the experiments and were killed after anaesthesia with ketamine and xylazine solution, 50 and $0.1 \mathrm{mg} / \mathrm{kg}$ respectively. One group of rats, which we called untreated, was divided into three subgroups: the first subgroup was given saline $(9 \mathrm{~g} \mathrm{NaCl} / 1)$ solution by oral administration. The second subgroup was given one of three concentrations of GSH $(0.4,1$ or $4 \mathrm{mmol} / \mathrm{kg})$, orally. The third subgroup was given a solution of $4 \mathrm{mmol}$ amino acids (Glu, Cys, Gly) $/ \mathrm{kg}$, orally. The rats were killed $90 \mathrm{~min}$ after these administrations. Another group of rats was treated with $\mathrm{AT}_{125}, 5.4 \mathrm{mmol} / \mathrm{kg}$ administered orally and $1.8 \mathrm{mmol} / \mathrm{kg}$ intraperitoneally; the administrations were performed at the same time. At 60 min after the $\mathrm{AT}_{125}$ treatment, one subgroup was given saline solution, orally; another subgroup was given $4 \mathrm{mmol}$ $\mathrm{GSH} / \mathrm{kg}$ orally. At $90 \mathrm{~min}$ after these administrations the rats were killed. A third group of rats was treated intraperitoneally twice, at 19.00 and 09.00 hours with a solution containing $4 \mathrm{mmol} \mathrm{BSO} / \mathrm{kg}$ and $150 \mathrm{ml}$ dimethylsulfoxide (DMSO) $/ 1$. At $30 \mathrm{~min}$ after the second BSO injection, one subgroup was given saline solution orally; a second subgroup was given one of two concentrations of GSH (either 0.4 or $4 \mathrm{mmol} / \mathrm{kg}$ ) orally; a third subgroup was given a solution containing $4 \mathrm{mmol}$ amino acids $/ \mathrm{kg}$ orally. At $90 \mathrm{~min}$ after these administrations the rats were killed. Since BSO is poorly transported into the brain of adult animals it was administered to rats intraperitoneally in $150 \mathrm{ml} \mathrm{DMSO} / 1$ which facilitates BSO absorption (Steinherz et al. 1990); for the same reason, the experiments were performed using 1month-old rats. Finally, under these experimental conditions, BSO treatment rapidly decreases the GSH levels in the organs studied, with GSH depletion values ranging from 40 to $90 \%$ of the control values. We also ascertained that intraperitoneal administration of $150 \mathrm{ml} \mathrm{DMSO} / 1$ solution alone did not change GSH levels in the various organs studied.

\section{Glutathione and $\gamma$-glutamyltranspeptidase assay}

After the various administrations, all organs of rats were removed and washed in ice-cold saline. Then they were frozen in liquid $\mathrm{N}_{2}$, pulverized and homogenized in $0.5 \mathrm{M}-\mathrm{HClO}_{4}$ $(1: 5, \mathrm{w} / \mathrm{v})$. The homogenate was centrifuged in a microcentrifuge $(12550 \mathrm{~g}$ for $15 \mathrm{~min}$ at $4^{\circ}$ ). The supernatant fraction was assayed for GSH and GSSG by HPLC as described by Fariss \& Reed (1987). The solutions were analysed by liquid chromatography (Beckman Gold System; Beckman Analytical S.p.A., Milan, Italy) equipped with an NEC/PC 8201H computer, a Shimadzu C-R6A chromatopac integrator (Shimadzu Corp., Kyoto, Japan), and a Biosil $\mathrm{NH}_{2}$ 90-5S Bio Rad column). The 2,4-dinitrophenyl derivatives were detected at $360 \mathrm{~nm}$. The GSH and GSSG were quantified relative to standards by integration and the values are expressed as $\mu \mathrm{mol} / \mathrm{g}$ wet weight. The total GSH, measured as (GSH $+2 \mathrm{GSSG}$ ), is reported.

$\gamma$-GT activity was measured in partially purified membranes of the organs studied. Using a Potter homogenizer (Wheaton Millville, NJ, USA) with a Teflon pestle, the intestinal mucosa and tissue samples of control and $\mathrm{AT}_{125}$-treated rats were homogenized in a buffer of ice-cold $100 \mathrm{~mm}$-Tris at $\mathrm{pH} 8(1: 6, \mathrm{w} / \mathrm{v})$. The homogenate was then 
centrifuged at $1900 \mathrm{~g}$ for $15 \mathrm{~min}$, and the supernatant fraction was centrifuged at $18000 \mathrm{~g}$ for $30 \mathrm{~min}$. Then the precipitate was collected, washed and centrifuged again; thereafter the pellet was used for the determination of $\gamma$-GT activity by a commercially available assay kit (Boehringer Mannheim GmbH, Mannheim, Germany).

\section{Protein determination}

The protein concentration was determined by Bradford's method (Bradford, 1976). Bovine serum albumin (Sigma Chemical Company) was used as the control standard.

\section{Statistical analyses}

The significance of treatment differences was tested using ANOVA. Student's $t$ test and the Mann-Whitney test. As the analyses gave similar results only the significance levels from the $t$ test are presented.

\section{RESULTS AND DISCUSSION}

GSH levels were measured in organs at various times after oral GSH administration $(60$, 90,120 and $180 \mathrm{~min}$ ). However, the data reported in Table 1 were determined $90 \mathrm{~min}$ after GSH administration, since at this time the highest GSH values were found in all organs studied; only in intestine was there no significant difference in GSH levels measured after 60 or 90 min.

Table 1 shows that oral administration of GSH to untreated rats and rats treated with BSO or $\mathrm{AT}_{125}$ significantly increased GSH content in jejunum of rats at all concentrations

Table 1. Glutathione (GSH) levels ( $\mu \mathrm{mol} / \mathrm{g}$ wet weight) in the jejunum, lung, liver, heart and brain of food-deprived rats $90 \mathrm{~min}$ after administration of saline solution, GSH or amino acids $(A A)$; rats had previously been untreated (control) or treated with L-buthionine sulfoximine (BSO) or acivicin $\left(A T_{125}\right)+$

(Mean values with their standard errors for six animals per group)

\begin{tabular}{|c|c|c|c|c|c|c|c|c|c|c|}
\hline \multirow[b]{2}{*}{ Treatment§ } & \multicolumn{2}{|c|}{ Jejunum } & \multicolumn{2}{|c|}{ Lung } & \multicolumn{2}{|c|}{ Heart } & \multicolumn{2}{|c|}{ Liver } & \multicolumn{2}{|c|}{ Brain } \\
\hline & Mean & SEM & Mean & SEM & Mean & SEM & Mean & SEM & Mean & SEM \\
\hline Control & 1.70 & 0.12 & 0.91 & 0.050 & 0.96 & 0.040 & 3.60 & 0.250 & 1.60 & 0.037 \\
\hline $0.4 \mathrm{GSH}$ & $2 \cdot 50^{*}$ & 0.34 & 1.00 & 0.160 & 1.00 & 0.040 & 3.80 & 0.900 & 1.50 & 0.124 \\
\hline $1 \mathrm{GSH}$ & $3.00^{*}$ & 0.20 & $1.25 *$ & $0 \cdot 110$ & $1.70 * *$ & 0.060 & $4 \cdot 50$ & 0.580 & 1.70 & 0.045 \\
\hline $4 \mathrm{GSH}$ & $6 \cdot 00^{* *}$ & 0.26 & $1 \cdot 34^{* *}$ & 0.088 & $1.73 * *$ & 0.060 & $5 \cdot 00 * *$ & 0.200 & $1.75^{*}$ & 0.036 \\
\hline BSO & $0.40^{* *}$ & 0.02 & $0.48^{* *}$ & 0.048 & $0.60^{* *}$ & 0.046 & $0.32 * *$ & 0.034 & $0.86^{* *}$ & 0.054 \\
\hline $\mathrm{BSO}+0.4 \mathrm{GSH}$ & $0.85 \dagger$ & 0.20 & $0.65 \dagger$ & 0.060 & $0.75 \dagger$ & 0.040 & 0.37 & 0.040 & 1.06 & 0.100 \\
\hline $\mathrm{BSO}+4 \mathrm{GSH}$ & $3.15+\dagger$ & 0.40 & $0.74+t$ & 0.046 & $0.80 \dagger$ & 0.035 & 0.36 & 0.027 & $1.05 \dagger$ & 0.035 \\
\hline $\mathrm{AT}_{125}$ & 1.45 & 0.12 & 0.97 & 0.068 & 1.00 & 0.100 & 3.33 & 0.400 & 1.61 & 0.090 \\
\hline $\mathrm{AT}_{125}+4 \mathrm{GSH}$ & $5 \cdot 80^{* *}$ & 0.50 & $1 \cdot 30^{* *}$ & 0.070 & $1.40^{* *}$ & 0.060 & 3.06 & 0.134 & 1.40 & 0.043 \\
\hline $\mathrm{AA}^{\|}$ & 1.90 & 0.06 & 1.13 & 0.058 & $1.40 * *$ & 0.035 & $5 \cdot 10^{*}$ & 0.600 & 1.68 & 0.098 \\
\hline $\mathrm{BSO}+\mathrm{AA}^{\| l}$ & & & & & 0.70 & 0.085 & 0.31 & 0.060 & & \\
\hline
\end{tabular}

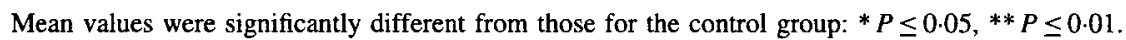

Mean values were significantly different from those for the BSO group: $+P \leq 0.05, \dagger+P \leq 0.01$.

$\ddagger$ For details of procedures, see pp. 294-296.

$\S \mathrm{GSH}$ was administered at levels of $0 \cdot 4,1$ and $4 \mathrm{mmol} / \mathrm{kg}$.

" AA (Glu, Cys, Gly) were administered at a level of $4 \mathrm{mmol} / \mathrm{kg}$. 
administered. In contrast, no significant alteration to GSH content was measured when an equivalent concentration of GSH constituent amino acids was given to untreated rats. The results indicate that in both untreated and treated rats increases in GSH level are not proportional to GSH concentrations. This is probably due to a saturation effect, as well as to a hormonal regulation of intestinal GSH absorption, as demonstrated by Hagen et al. (1991). The data obtained in jejunum of depleted rats are in agreement with the results reported in mice by Martensson et al. (1990) and Aw et al. (1991). These authors performed very different BSO treatments: Martensson et al. (1990) obtained a high GSH decrease (about $90 \%$ ), while Aw et al. (1991) obtained a decrease of only $20 \%$ and the GSH increase measured after oral administration of GSH was also different. However, these authors did not find GSH increases in jejunum of normal mice after oral GSH administration. This could be due to different experimental conditions, to the different species used, and to a different control of cellular GSH homeostasis in mice with respect to rats. However, Aw et al. (1991) showed a 2.5-fold increase in plasma GSH, $30 \mathrm{~min}$ after oral administration to mice of about $0.3 \mathrm{mmol} \mathrm{GSH} / \mathrm{kg}$; and Hagen et al. (1990) reported an increase of about 30-fold in plasma GSH level, 90 min after the administration of $30 \mu \mathrm{mol}$ GSH orally to BSO- and $\mathrm{AT}_{125}$-pretreated rats, while they did not observe any change when an equivalent amount of GSH constituent amino acids was given. Taken together with ours these results indicate that exogenous GSH is mainly absorbed intact in intestinal brush-border membranes by a transport system previously characterized by us (Vincenzini et al. 1988), confirming findings previously reported in part by the authors (Iantomasi et al. 1994). However breakdown of oral GSH by $\gamma$-GT intestinal activity may occur when high GSH concentrations are administered to rats. In fact, others (Viña et al. 1989) have measured increases in cysteine content in portal blood after the oral administration to rats of $3.26 \mathrm{mmol} \mathrm{GSH} / \mathrm{kg}$. In conclusion oral GSH may be useful in the treatment of many intestinal diseases and states of malnutrition, which can influence endogenous GSH by decreasing its synthesis, and/or GSH levels directly (Grisham, 1994; Iantomasi et al. 1994).

Table 1 shows that in lung and heart the picture obtained after oral administration of GSH to both untreated and treated rats was similar to that in intestine. One difference from intestine was the lack of increase in GSH content when the lower concentration of GSH $(0.4 \mathrm{mmol} / \mathrm{kg})$ was administered to untreated rats. A second difference was seen only in heart where a GSH increase was measured after amino acid administration to untreated rats compared with control. This last result indicates that GSH may be taken up by heart in part by transporter activity and in part by its degradation and subsequent resynthesis, while in lung, GSH absorption seems to occur as it does in intestine. Indeed, Hagen et al. (1986) showed that exogenous GSH may be taken up in lung alveolar cells by a $\mathrm{Na}^{+}$-dependent transport system, while there are no data on such a GSH transport system in heart membranes, even if the present results may suggest its presence. These findings are in agreement with the data reported by Aw et al. (1991), who found increased levels of lung GSH in untreated and BSO-treated animals at 90 and 60 min respectively after oral GSH administration. In contrast Martensson et al. (1989) measured only a transient GSH increase (of about $30 \%$ ) in lung of untreated mice $120 \mathrm{~min}$ after intraperitoneal GSH administration $(5 \mathrm{mmol} / \mathrm{kg})$ but not in depleted mice and did not measure any GSH increase in mouse heart after GSH depletion and intraperitoneal GSH administration. These contradictory data may be due to different experimental conditions and/or to the different species used. There are no data in the literature regarding the influence of $\gamma$-GT activity, determined by $\mathrm{AT}_{125}$ experiments, in increasing GSH content in organs after oral GSH administration. Under our present experimental conditions $\mathrm{AT}_{125}$ treatment completely 
inhibited $\gamma$-GT activity in intestine but only inhibited it by about $60 \%$ in lung (results not shown), even though $\gamma$-GT activity in lung was much lower than that measured in intestine, as also reported by others (Martensson et al. 1989; Bray \& Taylor, 1993). $\gamma$-GT activity was not detectable in heart membranes by the assay we used; Martensson \& Meister (1989) also found very low $\gamma$-GT activity in heart. Considering, therefore, the low activity of $\gamma$-GT in heart, we think that a GSH increase in heart may be in part due to the greater content of cysteine derived from the partial breakdown of oral GSH by intestinal $\gamma$-GT activity; this larger content of cysteine may then activate GSH synthesis. Indeed, in this organ, as in liver, GSH synthesis is mainly limited by the availability of cysteine (Martensson \& Meister, 1989). In conclusion, oral GSH could be clinically relevant in some heart pathologies and when increased oxidative stress is implicated, as during lung anti-tumour or $\mathrm{O}_{2}$ therapy (Witschi \& Lindenschmidt, 1985) and also in acquired immune deficiency syndrome patients, given their high susceptibility to lung infections (Stover et al. 1985). Table 1 also shows the data obtained in liver and brain. Significant increases in GSH content of liver with respect to controls were obtained only when $4 \mathrm{mmol} \mathrm{GSH} / \mathrm{kg}$ or an equivalent concentration of three amino acids were administered to untreated rats. These data are in agreement with the results obtained by Viña et al. (1989) and Aw et al. (1991) and show that the oral administration of a high dose of GSH $(4 \mathrm{mmol} / \mathrm{kg})$ may increase hepatic GSH content but only by degradation of GSH and subsequent intracellular resynthesis since no GSH increase was measured when GSH was administered to BSOand $\mathrm{AT}_{125}$-treated rats. However, considering that $\gamma$-GT activity of the liver in rat and mice is negligible, as also reported by Martensson et al. 1989, and that intraperitoneal administration of GSH is ineffective in increasing GSH level (Puri \& Meister, 1983), we suggest that, as for heart, the partial degradation of oral GSH obtained by the intestinal activity of $\gamma$-GT may supply the liver with cysteine and so increase GSH synthesis. It is probable that in a similar way oral GSH is able to increase GSH levels also in other tissues that do not have $\gamma$-GT activity and that do not take up GSH directly. Indeed two specific GSH transport systems in canalicular and sinusoidal membranes of hepatocytes have been found by Garcia-Ruiz et al. (1992). It has been suggested that in physiological conditions both transport systems mainly export GSH into bile and plasma. Our present results confirm this physiological role of hepatic GSH transporters; in fact, even if a remarkable GSH depletion in liver (about $90 \%$ ) is measured, no uptake of intact GSH seems to occur.

The findings obtained in the brain showed that a significant GSH increase was observed only when $4 \mathrm{mmol} \mathrm{GSH} / \mathrm{kg}$ was given to untreated and BSO-treated rats. These results are partly in agreement with those of others (Aw et al. 1991), who found similar GSH increases only in brain of GSH-depleted mice after GSH oral administration. The present results indicate that GSH is probably taken up intact by a transport system, although the lack of GSH increase in $\mathrm{AT}_{125}$-treated rats could indicate an involvement of $\gamma$ GT activity. Indeed, there is evidence that GSH may cross the blood-brain barrier by carrier-mediated transport (Kanna et al. 1990). Recently, Yi et al. (1994) demonstrated that complementary DNA of the rat canalicular GSH transporter hybridizes with a single transcript isolated from rat brain, and identified by Northern blot analysis. These authors also reported a similar single hybridization signal with RNA isolated from other organs: kidney, lung and small intestine. This evidence confirms the presence of similar transporters for GSH in different organs in which GSH levels may effectively increase by uptake of intact GSH. The present results demonstrate that exogenous GSH (present in bile, diet or drugs) may increase the GSH content of intestine and of some other organs, both GSH-depleted and -replete; they demonstrate that GSH intestinal absorption by $\gamma$-GT activity may be more useful in increasing GSH content in some organs (liver and heart) 
than intraperitoneal GSH administration. Moreover, these data demonstrate that both the specific transport systems of GSH, and $\gamma$-GT activity may have a physiological role in regulating GSH inter-organ homeostasis; they also confirm in part other data reported in the literature on this subject which is very important and complex, considering the various biological roles of GSH and its involvement in many pathological and physiological alterations.

This work was supported by grants from MURST, CNR and AIRC. We are grateful to Prof. G. Ramponi for his stimulating discussion and suggestions during the preparation of this manuscript.

\section{REFERENCES}

Aw, T. Y., Wierzbicka, G. \& Jones, D. P. (1991). Oral glutathione increases tissue glutathione in vivo. ChemicoBiological Interactions 80, 89-97.

Bradford, M. M. (1976). A rapid and sensitive method for the determination of microgram quantities of protein utilizing the principle of protein-dye binding. Analytical Biochemistry 72, 248-254.

Bray, T. M. \& Taylor, C. G. (1993). Tissue glutathione, nutrition, and oxidative stress. Canadian Journal of Physiology and Pharmacology 71, 746-751.

Castagna, A., Le Grazie, C., Accordini, A., Giulidori, P., Cavalli, G., Bottiglieri, T. \& Lazzarin, A. (1995). Cerebrospinal fluid S-adenosylmethionine (SAMe) and glutathione concentrations in HIV infection: effect of parenteral treatment with SAMe. Neurology 45, 1678-1683.

Fariss, M. W. \& Reed, D. J. (1987). High-performance liquid chromatography of thiols and disulfides: dinitrophenol derivatives. Methods in Enzymology 143, 101-109.

Favilli, F., Iantomasi, T., Marraccini, P., Stio, M., Lunghi, B., Treves, C. \& Vincenzini, M. T. (1994). Relationship between age and GSH metabolism in synaptosomes of rat cerebral cortex. Neurobiology of Aging 15, 429-433.

Garcia-Ruiz, C., Fernandez-Checa, J. \& Kaplowitz, N. (1992). Bidirectional mechanism of plasma membrane transport of reduced glutathione in intact rat hepatocytes and membrane vesicles. Journal of Biological Chemistry 267, 22256-22264.

Grisham, M. B. (1994). Oxidants and free radicals in inflammatory bowel disease. Lancet 344, 859-861.

Hagen, T. M., Brown, L. A. \& Jones, D. P. (1986). Protection against paraquat induced injury by exogenous GSH in pulmonary alveolar type II cells. Biochemical Pharmacology 35, 4537-4542.

Hagen, T. M., Wierzbicka, T. G., Sillau, A. H., Bowman, B. B. \& Jones, D. P. (1990). Bioavailability of dietary glutathione: effect on plasma concentration. American Journal of Physiology 259, G524-G529.

Hagen, T. M., Baj, C. \& Jones, D. P. (1991). Stimulation of glutathione absorption in rat small intestine by $\alpha-$ adrenergic agonists. FASEB Journal 5, 2721-2727.

Hill, K. E., Van Hoffand, D. D. \& Burk, R. F. (1985). Effect of inhibition of $\gamma$-glutamyltranspeptidase by AT $_{125}$ (Acivicin) on glutathione and cysteine levels in rat brain and plasma. Investigational New Drugs 3, 31-34.

Iantomasi, T., Marraccini, P., Favilli, F., Vincenzini, M. T., Ferretti, P. \& Tonelli, F. (1994). Glutathione metabolism in Crohn's disease. Biochemical Medicine and Metabolic Biology 53, 87-91.

Jones, D. P. (1995). Glutathione distribution in natural products: absorption and tissue distribution. In Methods in Enzymology, vol. 252, pp. 3-13 [L. Packer, editor]. New York: Academic Press.

Kanna, R., Kuhlenkamp, J., Jeandidier, E., Trinh, H., Ookhtens, M. \& Kaplowitz, N. (1990). Evidence for carrier-mediated transport of glutathione across the blood brain barrier in the rat. Journal of Clinical Investigation 85, 2009-2013.

Lash, L. H., Hagen, T. M. \& Jones, D. P. (1986). Exogenous glutathione protects intestinal epithelial cells from oxidative injury. Proceedings of the National Academy of Sciences USA 83, 4641- 4645.

Martensson, J., Jain, A., Frayer, W. \& Meister, A. (1989). Glutathione metabolism in the lung: inhibition of its synthesis leads to lamellar body and mitochondrial defects. Proceedings of the National Academy of Sciences USA 86, 5296-5300.

Martensson, J., Jain, A. \& Meister, A. (1990). Glutathione is required for intestinal function. Proceedings of the National Academy of Sciences USA 87, 1715-1719.

Martensson, J., Jain, A., Stole, E., Frayer, W., Auld, P. A. M. \& Meister, A. (1991). Inhibition of glutathione synthesis in the newborn rat: a model for endogenously produced oxidative stress. Proceedings of the National Academy of Sciences USA 88, 9360-9364.

Martensson, J. \& Meister, A. (1989). Mitochondrial damage in muscle occurs after marked depletion of glutathione and is prevented by giving glutathione monoester. Proceedings of the National Academy of Sciences USA 86, 471-475. 
Meister, A. \& Larsson, A. (1989). Glutathione synthetase deficiency and other disorders of the $\gamma$-glutamyl cycle. In The Metabolic Basis of Inherited Disease, pp. 855-868 [C. R. Scriver, A. L. Beaudet, W. S. Sly and D. Valle, editors]. New York: McGraw-Hill.

Puri, R. N. \& Meister, A. (1983). Transport of glutathione as $\gamma$-glutamylcysteinilglycyl ester, into liver and kidney. Proceedings of the National Academy of Sciences USA 80, 5258-5260.

Steinherz, R., Martensson, J., Wellner, D. \& Meister, A. (1990). Transport into brain of buthionine sulfoximine, an inhibitor of glutathione synthesis, is facilitated by esterification and administration of dimethylsulfoxide. Brain Research 518, 115-119.

Stio, M., Iantomasi, T., Favilli, F., Marraccini, P., Lunghi, B., Vincenzini, M. T. \& Treves, C. (1993). Glutathione metabolism in heart and liver of aging rat. Biochemistry and Cell Biology 72, 58-61.

Stover, D. E., White, D. A., Romano, T. A., Gellene, R. A. \& Robison, W. A. (1985). Spectrum of pulmonary diseases associated with the acquired immune deficiency syndrome. American Journal of Medicine 78, 429437.

Viña, J., Perez, C., Furukawa, T., Palacin, M. \& Viña, J. R. (1989). Effect of oral glutathione on hepatic glutathione levels in rats and mice. British Journal of Nutrition 62, 683-691.

Vincenzini, M. T., Favilli, F. \& Iantomasi, T. (1988). Glutathione mediated transport across intestinal brushborder membranes. Biochimica et Biophysica Acta 942, 107-114.

Vincenzini, M. T., Favilli, F. \& Iantomasi, T. (1992). Intestinal uptake and transmembrane transport systems of intact GSH; characteristics and possible biological role. Biochimica et Biophysica Acta 113, 13-23.

Witschi, H. P. \& Lindenschmidt, R. C. (1985). Pathogenesis of acute and chronic lung injury induced by foreign compounds. Clinical Physiology and Biochemistry 3, 135-146.

Yi, J. R., Lu, S., Fernandez-Checa, J. \& Kaplowitz, N. (1994). Expression cloning of a rat hepatic reduced glutathione transporter with canalicular characteristics. Journal of Clinical Investigation 93, 1841-1845. 